Pacific

Journal of

Mathematics

THE $K$-ORBIT OF A NORMAL ELEMENT IN A COMPLEX SEMISIMPLE LIE ALGEBRA

Tin-YaU TAM AND WaI-ShUn CheUnG

Volume $238 \quad$ No. 2

December 2008 


\title{
THE $K$-ORBIT OF A NORMAL ELEMENT IN A COMPLEX SEMISIMPLE LIE ALGEBRA
}

\author{
Tin-YAU TAM AND WAI-SHUn CHEUnG
}

\begin{abstract}
Given a complex semisimple $L i e$ algebra $\mathfrak{g}=\mathfrak{k}+i \mathfrak{k}$, we consider the converse question of Kostant's convexity theorem for a normal $x \in \mathfrak{g}$. Let $\pi: \mathfrak{g} \rightarrow \mathfrak{h}$ be the orthogonal projection under the Killing form onto the Cartan subalgebra $\mathfrak{h}:=\mathfrak{t}+i \mathfrak{t}$ where $\mathfrak{t}$ is a maximal abelian subalgebra of $\mathfrak{k}$. If $\pi(\operatorname{Ad}(K) x)$ is convex, then there is $k \in K$ such that each simple component of $\operatorname{Ad}(k) x$ can be rotated into the corresponding component of $t$. The result also extends a theorem of Au-Yeung and Tsing on the generalized numerical range.
\end{abstract}

\section{Introduction}

Let $A \in \mathbb{C}_{n \times n}$. Consider the set

$$
\mathcal{W}(A):=\left\{\operatorname{diag}\left(U A U^{-1}\right): U \in \mathrm{U}(n)\right\},
$$

where $\mathrm{U}(n)$ denotes the unitary group. It is the image of the projection of the orbit

$$
O(A):=\left\{U A U^{-1}: U \in \mathrm{U}(n)\right\}
$$

onto the set of diagonal matrices. The following two results concern the geometric shape of $\mathcal{W}(A)$.

Theorem 1.1 (Schur-Horn [Schur 1923; Horn 1954]). If $A \in \mathbb{C}_{n \times n}$ is Hermitian with eigenvalues $\lambda:=\left(\lambda_{1}, \ldots, \lambda_{n}\right) \in \mathbb{R}^{n}$, then

$$
\mathcal{W}(A)=\operatorname{conv} S_{n} \lambda,
$$

where conv $S_{n} \lambda$ is the convex hull of the orbit of $\lambda$ under the action of the full symmetric group $S_{n}$.

For general $A \in \mathbb{C}_{n \times n}, \mathcal{W}(A)$ is not convex. Indeed Tsing [1981] proved that $\mathcal{W}(A)$ is star-shaped with respect to the star center $\frac{1}{n}(\operatorname{tr} A)(1, \ldots, 1)$.

Theorem 1.2 (Au-Yeung and Sing [1977]). Let $A \in \mathbb{C}_{n \times n}$ be normal. If $W(A)$ is convex, then the eigenvalues of $A$ are collinear, that is, there exist $\alpha, \beta \in \mathbb{C}$ such that $\alpha A+\beta I$ is Hermitian.

MSC2000: primary 22E10; secondary 17B20.

Keywords: K-orbit, convex, normal element, complex semisimple Lie algebra. 
So Theorem 1.2 may be viewed as the converse to Theorem 1.1 as one restricts the attention on normal matrices. We remark that if $A \in \mathbb{C}_{n \times n}$ has zero trace, then $\alpha A+\beta I$ being Hermitian means that $e^{i \gamma} A$ is Hermitian for some $\gamma \in \mathbb{R}$. The following result of Au-Yeung and Tsing is stronger than Theorem 1.2. It affirmatively answers the conjecture of Marcus [1979] about the (stronger) converse of the result of Westwick [1975] on the convexity of $c$-numerical range. Bebiano and Da Providência [1996] gave another proof of Theorem 1.3.

Theorem 1.3 (Au-Yeung and Tsing [1983]). Let $A \in \mathbb{C}_{n \times n}$ be normal. If

$$
W_{A^{*}}(A):=\left\{\operatorname{tr} A^{*} U A U^{-1}: U \in \mathrm{U}(n)\right\}
$$

is convex, then A has collinear eigenvalues.

The above results can be reduced to the case $\operatorname{tr} A=0$, that is, the simple Lie algebra $\mathfrak{s l}_{n}(\mathbb{C})$. We may write $A=\hat{A}+\frac{1}{n}(\operatorname{tr} A) I_{n}$, where $\hat{A}:=A-\frac{1}{n}(\operatorname{tr} A) I_{n}$ has zero trace. Then

$$
\begin{aligned}
\mathscr{W}(A) & =\mathscr{W}(\hat{A})+\frac{\operatorname{tr} A}{n}(1, \ldots, 1), \\
W_{A^{*}}(A) & =W_{\hat{A}^{*}}(\hat{A})+\frac{|\operatorname{tr} A|^{2}}{n^{2}} .
\end{aligned}
$$

We will extend Theorems 1.2 and 1.3 in the context of semisimple Lie algebras.

\section{Main results}

Let $\mathfrak{g}$ be a complex semisimple Lie algebra and let $\mathfrak{k}$ be a real compact form of $\mathfrak{g}$. Let $G$ be a complex Lie group with Lie algebra $\mathfrak{g}$. It has a finite center so $K$ (the analytic group of $\mathfrak{k}$ ) is compact. As a real $K$-module, $\mathfrak{g}$ is just the direct sum of two copies of the adjoint module $\mathfrak{k}$ of $K: \mathfrak{g}=\mathfrak{k}+i \mathfrak{k}$ (direct sum), that is, Cartan decomposition of $\mathfrak{g}$. Denote by $\mathfrak{g}^{*}$ the dual space of $\mathfrak{g}$. Given $x \in \mathfrak{g}$, consider the orbit of $x$ under the adjoint action of $K$

$$
K \cdot x:=\{\operatorname{Ad}(k) x: k \in K\} .
$$

The orbit $K \cdot x$ depends on $\operatorname{Ad}_{G} K$ which is the analytic subgroup of the adjoint group $\operatorname{Int}(\mathfrak{g}) \subset$ Aut $(\mathfrak{g})$ corresponding to ad $\mathfrak{g}(\mathfrak{k})$. Thus $K \cdot x$ is independent of the choice of $G$. Let $\mathfrak{t}$ be a maximal abelian subalgebra of $\mathfrak{k}$. The complexification $\mathfrak{h}:=\mathfrak{t}+i \mathfrak{t}$ (direct sum) is a Cartan subalgebra of $\mathfrak{g}$. The rank of $\mathfrak{g}$ is $\operatorname{dim}_{\mathbb{C}} \mathfrak{h}$, denoted by rank $\mathfrak{g}$. Let

$$
\mathfrak{g}=\mathfrak{h}+\sum_{\alpha \in \Delta} \mathfrak{g}_{\alpha}
$$

be the root space decomposition of $\mathfrak{g}$ with respect to $\mathfrak{h}$, where $\Delta$ denotes the set of all nonzero roots. Denote by $B(\cdot, \cdot)$ the Killing form of $\mathfrak{g}$. As $B(\cdot, \cdot)$ is a nondegenerate bilinear form, it induces a vector space isomorphism $\mathfrak{g} \rightarrow \mathfrak{g}^{*}$ sending 
$x \rightarrow \varphi_{x}$, where $\varphi_{x}(y)=B(x, y)$ for all $y \in \mathfrak{g}$. Denote the inverse by $\varphi \rightarrow H_{\varphi} \in \mathfrak{g}$ $\left(\varphi \in \mathfrak{g}^{*}\right)$, where $B\left(H_{\varphi}, y\right)=\varphi(y)$ for all $y \in \mathfrak{g}$. Let

$$
\mathfrak{h}_{\mathbb{R}}:=\sum_{\alpha \in \Delta} \mathbb{R} H_{\alpha}
$$

so that $B(\cdot, \cdot)$ is a real inner product on $\mathfrak{h}_{\mathbb{R}}$ and $\mathfrak{h}=\mathfrak{h}_{\mathbb{R}}+i \mathfrak{h}_{\mathbb{R}}$ (direct sum). Hence rank $\mathfrak{g}=\operatorname{dim}_{\mathbb{R}} \mathfrak{h}_{\mathbb{R}}$. Moreover $\mathfrak{h}_{\mathbb{R}}=i \mathfrak{t}$ [Helgason 1978, p. 259]. Notice that $B\left(\mathfrak{g}_{\alpha}, \mathfrak{g}_{\beta}\right)=0$ [Helgason 1978, p. 166] whenever $\alpha+\beta \neq 0\left(\mathfrak{g}_{0}=\mathfrak{h}\right)$ so the sum

$$
\mathfrak{g}=\mathfrak{h}+\sum_{\alpha \in \Delta^{+}}\left(\mathfrak{g}_{\alpha}+\mathfrak{g}_{-\alpha}\right)
$$

is orthogonal under the Killing form. Thus we have the orthogonal projection $\pi: \mathfrak{g} \rightarrow \mathfrak{h}$ under $B(\cdot, \cdot)$. For $x \in \mathfrak{g}$, we consider $\pi(K \cdot x)$, that is, the projection of $K \cdot x$ onto $\mathfrak{h}$. When $x \in \mathfrak{k}, K \cdot x \subset \mathfrak{k}$ so $\pi(K \cdot x) \subset \mathfrak{t}$.

Kostant [1973] generalized Theorem 1.1 in the context of real semisimple Lie algebras. The following statement is for complex semisimple case. When $\mathfrak{g}=$ $\mathfrak{s l}_{n}(\mathbb{C})$, it is reduced to Theorem 1.1.

Theorem 2.1 (Kostant [1973]). If $x \in \mathfrak{k}$, then $\pi(K \cdot x) \subset \mathfrak{t}$ is convex and equals to conv $W x_{\mathfrak{t}}$, where $x_{\mathfrak{t}} \in K \cdot x \cap \mathfrak{t}$ and $W$ is the Weyl group, that is, $W=N(T) / T$, the normalizer of $T$ modulo $T$.

Let $\theta$ be the Cartan involution of $\mathfrak{g}$ if $\mathfrak{g}$ is viewed as a real Lie algebra, that is, $\theta: \mathfrak{g} \rightarrow \mathfrak{g}$ such that $x+y \mapsto x-y$ if $x \in \mathfrak{k}$ and $y \in i \mathfrak{k}$. In other words, $\mathfrak{k}$ is the +1 eigenspace of $\theta$ and $i \mathfrak{k}$ is the -1 eigenspace of $\theta$. Though $\theta$ is not an automorphism of $\mathfrak{g}$ over $\mathbb{C}$ (since $\theta(c x)=\bar{c} \theta x$ for $c \in \mathbb{C}$ and $x \in \mathfrak{g}$ ), it respects the bracket, that is,

$$
\theta[x, y]=[\theta x, \theta y], \quad x, y \in \mathfrak{g} .
$$

Moreover $\operatorname{Ad}(k)$ and $\theta$ commute for all $k \in K$. Since $\mathfrak{g}=\mathfrak{k}+i \mathfrak{k}$ and $\mathfrak{k}$ is compact,

$$
B_{\theta}(x, y):=-B(x, \theta y)
$$

is an inner product on $\mathfrak{g}$ over $\mathbb{C}$. Let

$$
\|x\|_{\theta}:=B_{\theta}^{1 / 2}(x, x)
$$

be the induced norm on $\mathfrak{g}$. The projection $\pi: \mathfrak{g} \rightarrow \mathfrak{h}$ under $B(\cdot, \cdot)$ coincides with that under $B_{\theta}(\cdot, \cdot)$ since $\theta \mathfrak{g}_{\alpha}=\mathfrak{g}_{-\alpha}$, for $\alpha \in \Delta$.

An element $x \in \mathfrak{g}$ is said to be normal if $[x, \theta x]=0$, where $\theta$ is the Cartan involution. When $\mathfrak{g}=\mathfrak{s l}_{n}(\mathbb{C})$, the Cartan decomposition is the usual Hermitian decomposition, $K=\mathrm{SU}(n)$ and $\theta(z)=-z^{*}, z \in \mathfrak{s l}_{n}(\mathbb{C})$. When $\mathfrak{g}=\mathfrak{s l}_{n}(\mathbb{C})$ and $\mathfrak{k}=\mathfrak{s u}(n)$, normality reduces to the usual notion of normality of a matrix. 
We want to know when $\pi(K \cdot x)$ is convex, that is, the converse question of Theorem 2.1 when we restrict ourselves to normal $x \in \mathfrak{g}$. Djoković and Tam [2003] proved that $B_{\theta}(K \cdot x, y) \subset \mathbb{C}$ is star shaped with respect the origin for each $y \in \mathfrak{g}$, if $x \in \mathfrak{g}$ is normal. In particular $B_{\theta}(K \cdot x, x)$ is star shaped. We also want to know when $B_{\theta}(K \cdot x, x)$ is convex. It turns out their answers coincide as suggested by Theorems 1.2 and 1.3. Indeed it is equivalent to say that $B_{\theta}(K \cdot x, y)$ is convex for all $y \in \mathfrak{g}$ in the following theorem.

Theorem 2.2. Let $\mathfrak{g}=\mathfrak{g}_{1}+\cdots+\mathfrak{g}_{\ell}$ be a complex semisimple Lie algebra with simple components $\mathfrak{g}_{1}, \ldots, \mathfrak{g}_{\ell}$. Let $x=x_{1}+\cdots+x_{\ell} \in \mathfrak{g}$ be normal, where $x_{i} \in \mathfrak{g}_{i}$, $i=1, \ldots, \ell$. The following statements are equivalent:

(1) $\pi(K \cdot x)$ is convex.

(2) $B_{\theta}(K \cdot x, x)$ is convex.

(3) $B_{\theta}(K \cdot x, x)$ is a closed line segment in $\mathbb{R}$.

(4) $K_{j} \cdot e^{i \theta_{j}} x_{j} \cap \mathfrak{t}_{j}$ is nonempty for some $\theta_{j} \in[0,2 \pi], j=1, \ldots, \ell$.

(5) $B_{\theta}(K \cdot x, y)$ is convex for all $y \in \mathfrak{g}$.

Remark 2.3. Normality of $x \in \mathfrak{g}$ is necessary. When $\mathfrak{g}=\mathfrak{s l}_{n}(\mathbb{C})$ and $K=\mathrm{SU}(n)$, it is known that $B_{\theta}(K \cdot x, y)$ is convex for all $y \in \mathfrak{s l}_{n}(\mathbb{C})$ if $x \in \mathfrak{s l}_{n}(\mathbb{C})$ and the matrix rank of $x$ is 1 (not necessarily normal), according to a result of Tsing [1984]. For example, if

$$
x=\left(\begin{array}{ll}
0 & 1 \\
0 & 0
\end{array}\right) \oplus 0_{n-2},
$$

then $B_{\theta}(K \cdot x, y)$ is convex for all $y \in \mathfrak{s l}_{n}(\mathbb{C})$. However statement (3) in Theorem 2.2 does not hold.

We first establish some results in order to prove Theorem 2.2.

A line $L$ is called a support of $B_{\theta}(K \cdot x, x) \subset \mathbb{C}$ at $\xi \in \partial B_{\theta}(K \cdot x, x)$ if $B_{\theta}(K \cdot x, x)$ lies in one of the closed half planes determined by $L$. A point $\xi \in B_{\theta}(K \cdot x, x)$ is called an extreme point of $B_{\theta}(K \cdot x, x)$ if $\xi$ does not belong to any open line segment lying in $B_{\theta}(K \cdot x, x)$. It is clear that extreme points belong to $\partial B_{\theta}(K \cdot x, x)$. An extreme point $\xi \in B_{\theta}(K \cdot x, x)$ is called a sharp point if $B_{\theta}(K \cdot x, x)$ has more than one support line at $\xi$. Clearly a sharp point $\xi$ of $B_{\theta}(K \cdot x, x)$ is an extreme point. The definitions are valid for convex sets in $\mathbb{C}$. The notions of extreme point and sharp point of a convex polygon in $\mathbb{C}$ coincide. We remark that $B_{\theta}(K \cdot x, x)$ is not necessarily a convex polygon.

Proposition 2.4. Let $x \in \mathfrak{g}$ be normal.

(a) $B_{\theta}(K \cdot x, x) \subset \mathbb{C}$ is symmetric about the real axis. 
(b) $B_{\theta}(K \cdot x, x) \subset \mathbb{C}$ is contained in the convex polygon

$$
B_{\theta}\left(\operatorname{conv} W x_{1}+i \operatorname{conv} W x_{2}, x\right)
$$

where $x=x_{1}+i x_{2}, x_{1}, x_{2} \in \mathfrak{k}$. Both sets contain the point $B_{\theta}(x, x) \geq 0$ which has the largest magnitude. Thus $B_{\theta}(x, x)$ is a sharp point of both $B_{\theta}(K \cdot x, x)$ and $B_{\theta}\left(\operatorname{conv} W x_{1}+i \operatorname{conv} W x_{2}, x\right)$.

Proof. Since $\theta$ and $\operatorname{Ad}(k)(k \in K)$ commute, for $x, y \in \mathfrak{g}$,

$$
B_{\theta}(\operatorname{Ad}(k) x, \operatorname{Ad}(k) y)=-B(\operatorname{Ad}(k) x, \operatorname{Ad}(k) \theta y)=B_{\theta}(x, y)
$$

and hence $\operatorname{Ad}(k): \mathfrak{g} \rightarrow \mathfrak{g}$ is an isometry with respect to $B_{\theta}(\cdot, \cdot)$.

(a) Let $x \in \mathfrak{g}$ be normal. Clearly

$$
\overline{B_{\theta}(\operatorname{Ad}(k) x, x)}=B_{\theta}(x, \operatorname{Ad}(k) x)=B_{\theta}\left(\operatorname{Ad}\left(k^{-1}\right) x, x\right) .
$$

Hence (a) is established.

(b) Since $x=x_{1}+i x_{2} \in \mathfrak{g}\left(x_{1}, x_{2} \in \mathfrak{k}\right)$ is normal, $K \cdot x$ intersects $\mathfrak{h}$ [Djoković and Tam 2003, Lemma 3.3.14]. So we may assume that $x_{1}, x_{2} \in \mathfrak{t}$. By Theorem 2.1

$$
\begin{aligned}
\pi(K \cdot x) & =\pi\left(K \cdot\left(x_{1}+i x_{2}\right)\right) \\
& \subset \pi\left(K \cdot x_{1}+i K \cdot x_{2}\right) \\
& =\pi\left(K \cdot x_{1}\right)+i \pi\left(K \cdot x_{2}\right) \\
& =\operatorname{conv} W x_{1}+i \operatorname{conv} W x_{2},
\end{aligned}
$$

where the sum conv $W x_{1}+i$ conv $W x_{2}$ is a convex polytope in $\mathfrak{h}$. Since $\pi: \mathfrak{g} \rightarrow \mathfrak{h}$ is also an orthogonal projection with respect to $B_{\theta}(\cdot, \cdot)$,

$$
B_{\theta}(K \cdot x, x)=B_{\theta}(\pi(K \cdot x), x)
$$

is contained in the convex polygon $B_{\theta}\left(\operatorname{conv} W x_{1}+i \operatorname{conv} W x_{2}, x\right)$. Let

$$
y \in \operatorname{conv} W x_{1} \subset \mathfrak{t} \quad \text { and } \quad z \in \operatorname{conv} W x_{2} \subset \mathfrak{t} .
$$

Since $\mathfrak{h}_{\mathbb{R}}:=\sum_{\alpha \in \Delta} \mathbb{R} H_{\alpha}=i t$ [Helgason 1978, p. 259] and $\alpha(H) \in \mathbb{R}$ for each $H \in \mathfrak{h}_{\mathbb{R}}, \alpha \in \Delta, \alpha(y), \alpha\left(x_{1}\right) \in i \mathbb{R}$ and $\alpha(i z), \alpha\left(i x_{2}\right) \in \mathbb{R}$. Hence

$$
\alpha(\theta x)=-\overline{\alpha(x)}
$$

SO

$$
\|x\|_{\theta}^{2}=B_{\theta}(x, x)=\sum_{\alpha \in \Delta}|\alpha(x)|^{2} .
$$

Moreover

$$
\|y+i z\|_{\theta}^{2}=\sum_{\alpha \in \Delta} \alpha(y+i z) \overline{\alpha(y+i z)}=\sum_{\alpha \in \Delta}\left(|\alpha(y)|^{2}+|\alpha(i z)|^{2}\right)=\|y\|_{\theta}^{2}+\|i z\|_{\theta}^{2} .
$$


By Cauchy-Schwarz's inequality

$$
\left|B_{\theta}(y+i z, x)\right|^{2} \leq\|y+i z\|_{\theta}^{2}\|x\|_{\theta}^{2}=\left(\|y\|_{\theta}^{2}+\|i z\|_{\theta}^{2}\right)\|x\|_{\theta}^{2} .
$$

Using triangle inequality, we have

$$
\|y\|_{\theta}^{2} \leq\left\|x_{1}\right\|_{\theta}^{2}, \quad\|i z\|_{\theta}^{2} \leq\left\|i x_{2}\right\|_{\theta}^{2},
$$

since the elements in $W$ are isometries. By (2-1) and (2-2)

$$
\left|B_{\theta}(y+i z, x)\right|^{2} \leq B_{\theta}^{2}(x, x) .
$$

Remark 2.5. Given $x \in \mathfrak{h}, W x \subset K \cdot x$ and thus $W x \subset \pi(K \cdot x)$. We do not know whether $\pi(K \cdot x) \subset$ conv $W x$ or not though it is true when $\mathfrak{g}=\mathfrak{s l}_{n}(\mathbb{C})$.

Lemma 2.6. Let $x \in \mathfrak{g}$ be normal. Then $B_{\theta}(K \cdot x, x)$ is convex if and only if it is a closed interval in $\mathbb{R}$.

Proof. One implication is trivial. Suppose $B_{\theta}(K \cdot x, x)$ is convex and we may assume $x \neq 0$. By Proposition 2.4

$$
\xi:=B_{\theta}(x, x)=\|x\|_{\theta}
$$

is a sharp point of $B_{\theta}(K \cdot x, x)$. There are two supporting lines passing through $\xi$ and one is the reflection of the other by Proposition 2.4 (a). Clearly $B_{\theta}(K \cdot x, x)$ is inside the cone determined by the two lines. Let $L$ be the upper supporting line for definiteness. So $B_{\theta}(K \cdot x, x)$ is in the lower half plane determined by $L$.

By [Djoković and Tam 2003, Lemma 3.14] we may assume that $x=x_{1}+i x_{2} \in$ $\mathfrak{h}, x_{1}, x_{2} \in \mathfrak{t}$. Let $\xi_{j}:=B_{\theta}\left(\operatorname{Ad}\left(k_{j}\right) x, x\right)\left(k_{j} \in K\right)$ be on the upper boundary of $B_{\theta}(K \cdot x, x)$ so that $\left|\xi-\xi_{j}\right|<1 / j$ but $\xi_{j} \neq \xi, j=1,2, \ldots$ Since $K$ is compact, there is a convergent subsequence $\left\{k_{j_{m}}\right\}_{m=1}^{\infty}$ of $\left\{k_{j}\right\}_{j=1}^{\infty}$. Let $\lim _{m \rightarrow \infty} k_{j_{m}}=k_{0} \in K$. So

$$
B_{\theta}\left(\operatorname{Ad}\left(k_{0}\right) x, x\right)=\xi=B_{\theta}(x, x)=\left\|\operatorname{Ad}\left(k_{0}\right) x\right\|_{\theta}\|x\|_{\theta}
$$

since $\operatorname{Ad}\left(k_{0}\right)$ is an isometry. By the equality case of Cauchy-Schwarz's inequality, $\operatorname{Ad}\left(k_{0}\right) x=x$. Thus

$$
B_{\theta}\left(\operatorname{Ad}\left(k_{j}\right) x, x\right)=B_{\theta}\left(\operatorname{Ad}\left(k_{j}\right) x, \operatorname{Ad}\left(k_{0}\right) x\right)=B_{\theta}\left(\operatorname{Ad}\left(k_{0}^{-1} k_{j}\right) x, x\right) .
$$

We may replace $k_{j_{m}}$ by $k_{0}^{-1} k_{j_{m}} \rightarrow e$ (the identity) or simply assume that $k_{0}=e$. The exponential map is an analytic diffeomorphism between an open neighborhood of $0 \in \mathfrak{k}$ and an open neighborhood of $e \in K$. So for each sufficiently large $m$, there is $s_{j_{m}} \in \mathfrak{k}$ such that

$$
\exp s_{j_{m}}=k_{j_{m}} \rightarrow e
$$


Since $x \in \mathfrak{h}$,

$$
\begin{aligned}
\xi_{j_{m}}= & B_{\theta}\left(\operatorname{Ad}\left(e^{s_{j_{m}}}\right) x, x\right)=B_{\theta}\left(e^{\operatorname{ad} s_{j_{m}}} x, x\right) \\
= & B_{\theta}(x, x)+B_{\theta}\left(\operatorname{ad} s_{j_{m}} x, x\right)+\frac{1}{2} B_{\theta}\left(\left(\operatorname{ad} s_{j_{m}}\right)^{2} x, x\right) \\
& +\sum_{k=3}^{\infty} \frac{1}{k !} B_{\theta}\left(\left(\operatorname{ad} s_{j_{m}}\right)^{k} x, x\right) .
\end{aligned}
$$

The first term of (2-3) is just $\xi$. The second term is

$$
-B\left(\operatorname{ad}\left(s_{j_{m}}\right) x, \theta x\right)=-B\left(\left[s_{j_{m}}, x\right], \theta x\right)=-B\left(s_{j_{m}},[x, \theta x]\right)=0,
$$

because $[x, \theta x]=0$. Since the elements in ad $\mathfrak{k}$ are skew Hermitian with respect to $B_{\theta}(\cdot, \cdot)$, the third term is

$$
B_{\theta}\left(\left(\operatorname{ad} s_{j_{m}}\right)^{2} x, x\right)=-B_{\theta}\left(\operatorname{ad} s_{j_{m}} x, \operatorname{ad}\left(s_{j_{m}}\right) x\right)=-\left\|\operatorname{ad}\left(s_{j_{m}}\right) x\right\|_{\theta}^{2} .
$$

Taking the absolute value of the last term of (2-3), we have

$$
\begin{aligned}
\left|\sum_{k=3}^{\infty} \frac{1}{k !} B_{\theta}\left(\left(\operatorname{ad} s_{j_{m}}\right)^{k} x, x\right)\right| & =\left|\sum_{k=3}^{\infty} \frac{1}{k !} B_{\theta}\left(\operatorname{ad} s_{j_{m}} \circ\left(\operatorname{ad} s_{j_{m}}\right)^{k-2} \circ \operatorname{ad}\left(s_{j_{m}}\right) x, x\right)\right| \\
& =\sum_{k=3}^{\infty} \frac{1}{k !}\left|B_{\theta}\left(\left(\operatorname{ad} s_{j_{m}}\right)^{k-2} \circ \operatorname{ad}\left(s_{j_{m}}\right) x, \operatorname{ad}\left(s_{j_{m}}\right) x\right)\right| \\
& \leq \sum_{k=3}^{\infty} \frac{1}{k !}\left\|\left(\operatorname{ad} s_{j_{m}}\right)^{k-2} \operatorname{ad}\left(s_{j_{m}}\right) x\right\|_{\theta}\left\|\operatorname{ad}\left(s_{j_{m}}\right) x\right\|_{\theta} \\
& \leq \sum_{k=3}^{\infty} \frac{1}{k !}\left\|\left(\operatorname{ad} s_{j_{m}}\right)^{k-2}\right\|_{\theta}\left\|\operatorname{ad}\left(s_{j_{m}}\right) x\right\|_{\theta}^{2} \\
& \leq\left(e^{\left\|\operatorname{ad} s_{j_{m}}\right\|}-1\right)\left\|\operatorname{ad}\left(s_{j_{m}}\right) x\right\|_{\theta}^{2},
\end{aligned}
$$

where

$$
\left\|\operatorname{ad} s_{j_{m}}\right\|:=\max _{y \in \mathfrak{g},\|y\|_{\theta}=1}\left\|\operatorname{ad}\left(s_{j_{m}}\right) y\right\|_{\theta}
$$

is the operator norm of ad $s_{j_{m}}: \mathfrak{g} \rightarrow \mathfrak{g}$ with respect to $\|\cdot\|_{\theta}$. Notice that ad $\left(s_{j_{m}}\right) x \neq 0$ otherwise $\xi=\xi_{j_{m}}$ from (2-3). Since $s_{j_{m}} \rightarrow 0\left(x \neq 0, s_{j_{m}} \neq 0\right)$,

$$
\lim _{m \rightarrow \infty} \frac{\left|\sum_{k=3}^{\infty} \frac{1}{k !} B_{\theta}\left(\left(\operatorname{ad} s_{j_{m}}\right)^{k} x, x\right)\right|}{\left\|\operatorname{ad}\left(s_{j_{m}}\right) x\right\|_{\theta}^{2}}=0 .
$$

Consequently we have

$$
\lim _{m \rightarrow \infty} \frac{\xi-\xi_{j_{m}}}{\left\|\operatorname{ad}\left(s_{j_{m}}\right) x\right\|_{\theta}^{2}}=\frac{1}{2}
$$


Since $B_{\theta}(K \cdot x, x)$ is convex, there is $\xi^{\prime} \in L \cap \partial B_{\theta}(K \cdot x, x)$ so that the line segment $\left[\xi, \xi^{\prime}\right] \subset \partial B_{\theta}(K \cdot x, x)$. For sufficiently large $m, \xi_{j_{m}} \in\left[\xi, \xi^{\prime}\right]$, thus the limit on the left-hand side of (2-4) must be a positive multiple of $\xi-\xi^{\prime}$. So $\xi^{\prime} \in \mathbb{R}$ and thus $L \subset \mathbb{R}$. Therefore the compact connected set $B_{\theta}(K \cdot x, x)$ is a closed interval in $\mathbb{R}$.

Proposition 2.7. Let $\mathfrak{g}=\mathfrak{g}_{1}+\cdots+\mathfrak{g}_{\ell}$ be a complex semisimple Lie algebra with simple components $\mathfrak{g}_{1}, \ldots, \mathfrak{g}_{\ell}$. Let $x, y \in \mathfrak{h}:=\mathfrak{t}+i \mathfrak{t}$. Write $x=x_{1}+\cdots+x_{\ell}$ and $y=y_{1}+\cdots+y_{\ell}$, where $x_{i}, y_{i} \in \mathfrak{h}_{i}, i=1, \ldots, \ell$. Suppose that $x_{i}, y_{i}$ are nonzero for all $i=1, \ldots, \ell$. Then the following statements are equivalent.

(1) $B_{\theta}(K \cdot x, y)$ is a (closed) line segment in $\mathbb{C}$.

(2) $B_{\theta}(W \cdot x, y)$ is on a line segment in $\mathbb{C}$, where $W$ is the Weyl group.

(3) $K_{j} \cdot e^{i \theta_{j}} x_{j} \cap \mathfrak{t}_{j}$ and $K_{j} \cdot e^{i \rho_{j}} y_{j} \cap \mathfrak{t}_{j}$ are nonempty for some $\theta_{j}, \rho_{j} \in[0,2 \pi]$, $j=1, \ldots, \ell$, and $\kappa:=\theta_{j}-\rho_{j}$ is a constant for all $j=1, \ldots, \ell$.

Proof. (1) $\Rightarrow$ (2) is trivial.

(3) $\Rightarrow(1)$ : We may assume that $e^{i \theta_{j}} x_{j} \in \mathfrak{t}_{j}$ and $e^{i \rho_{j}} x_{j} \in \mathfrak{t}_{j}$ since

$$
B_{\theta}(K \cdot x, y)=B_{\theta}(K \cdot x, K \cdot y) .
$$

Now

$$
\begin{aligned}
B_{\theta}(K \cdot x, y) & =B_{\theta}\left(K_{1} \cdot x_{1}, y_{1}\right)+\cdots+B_{\theta}\left(K_{\ell} \cdot x_{\ell}, y_{\ell}\right) \\
& =e^{-i \kappa} \sum_{j=1}^{\ell} B_{\theta}\left(K_{1} \cdot e^{i \theta_{j}} x_{j}, e^{i \rho_{j}} y_{j}\right)
\end{aligned}
$$

and each summand $B_{\theta}\left(K_{1} \cdot e^{i \theta_{j}} x_{j}, e^{i \rho_{j}} y_{j}\right) \subset \mathbb{R}$.

$(2) \Rightarrow(3)$ : Suppose $B_{\theta}(W x, y)$ is a (closed) line segment. By rotation on $x$ or $y$ we may assume that $B_{\theta}(W x, y) \subset \mathbb{R}$. Since

$$
B_{\theta}(W x, y)=B_{\theta}\left(W_{1} x_{1}, y_{1}\right)+\cdots+B_{\theta}\left(W_{\ell} x_{\ell}, y_{\ell}\right),
$$

each $B_{\theta}\left(W_{j} x_{j}, y_{j}\right)$ is a real line segment, $j=1, \ldots, \ell$. So it suffices to consider simple $\mathfrak{g}_{j}$. To simplify notations, from now on we drop the index $j$ from $\mathfrak{g}_{j}, \mathfrak{k}_{j}, \mathfrak{t}_{j}$, $\mathfrak{h}_{j}, x_{j}, r_{j}$ and so on, or simply assume that $\mathfrak{g}$ is simple.

Notice that

$$
\tau_{H_{\beta}}\left(H_{\alpha}\right)=H_{\alpha}-\frac{2 B\left(H_{\alpha}, H_{\beta}\right)}{B\left(H_{\beta}, H_{\beta}\right)} H_{\beta}, \quad \alpha, \beta \in \Delta .
$$

As a finite reflection group, the Weyl group $W$ is generated by the reflections $\tau_{H_{\beta}}$, $\beta \in \Delta$, and

$$
B_{\theta}\left(W x, \tau_{H_{\beta}} y\right)=B_{\theta}(W x, y) \subset \mathbb{R}
$$


so for all $\omega \in W$ and $\beta \in \Delta$,

$$
\begin{aligned}
B_{\theta}\left(\omega x, \tau_{H_{\beta}} y\right) & =B_{\theta}\left(\omega x, y-\frac{2 \beta(y)}{\|\beta\|_{\beta}^{2}} H_{\beta}\right) \\
& =B_{\theta}(\omega x, y)-\frac{2 \overline{\beta(y)}}{\|\beta\|_{\beta}^{2}} B_{\theta}\left(\omega x, H_{\beta}\right) .
\end{aligned}
$$

Hence for all $\beta \in \Delta$,

$$
\frac{2 \overline{\beta(y)}}{\|\beta\|_{\theta}^{2}} B_{\theta}\left(W x, H_{\beta}\right) \subset \mathbb{R}
$$

so either (a) $B_{\theta}\left(H_{\beta}, y\right)=\beta(y)=0$ for all $\beta \in \Delta$, or (b) for some $\beta \in \Delta$ (depends on $y), \beta(y) \neq 0$, that is, $e^{i \gamma} B_{\theta}\left(W x, H_{\beta}\right) \subset \mathbb{R}$ for some $\gamma \in \mathbb{R}$.

Since $\mathfrak{h}=\sum_{\beta \in \Delta} \mathbb{C} H_{\beta}$ and $B$ is nondegenerate on $\mathfrak{h}$, (a) would not occur because we assume that $y \neq 0$. So (b) occurs, that is, $B_{\theta}\left(W e^{i \gamma} x, H_{\beta}\right) \subset \mathbb{R}$. But then

$$
B_{\theta}\left(W H_{\beta}, e^{i \gamma} x\right)=\overline{B_{\theta}\left(W e^{i \gamma} x, H_{\beta}\right)} \subset \mathbb{R} .
$$

Similarly for all $\alpha \in \Delta$,

$$
\frac{2 \overline{\alpha\left(e^{i \gamma} x\right)}}{\left\|H_{\alpha}\right\|_{\theta}^{2}} B_{\theta}\left(W H_{\beta}, H_{\alpha}\right) \subset \mathbb{R} .
$$

Now $B_{\theta}\left(W H_{\beta}, H_{\alpha}\right) \subset \mathbb{R}$ since $H_{\alpha}, H_{\beta} \in \mathfrak{h}_{\mathbb{R}}=i$ t. By contragradience the Weyl group permutes the roots. If $\omega \in W$ then $\omega H_{\beta}=H_{\omega \cdot \beta}$. We claim that

$$
B_{\theta}\left(\omega H_{\beta}, H_{\alpha}\right) \neq 0 \text {, for some } \omega \in W \text {. }
$$

It is because that the Weyl group acts simply transitively on each subset of roots of the same length [Helgason 1978, p. 523]. If $H_{\alpha}$ and $H_{\beta}$ are of the same length, then $\omega H_{\beta}=H_{\alpha}$ for some $\omega \in W$ and $B_{\theta}\left(\omega H_{\beta}, H_{\alpha}\right)=\left\|H_{\alpha}\right\|_{\theta}^{2}>0$. Hence the claim follows immediately. When $\mathfrak{g}=\mathfrak{a}_{n}, \mathfrak{d}_{n}, \mathfrak{e}_{6}, \mathfrak{e}_{7}, \mathfrak{e}_{8}$, all the roots are of the same length [Helgason 1978, p. 462-474]. Notice that

$$
\begin{aligned}
\mathfrak{b}_{n}: \Delta & =\left\{ \pm e_{i} \pm e_{j}: 1 \leq i \neq j \leq n\right\} \cup\left\{ \pm e_{i}: 1 \leq i \leq n\right\}, \\
\mathfrak{c}_{n}: \Delta & =\left\{ \pm e_{i} \pm e_{j}: 1 \leq i \neq j \leq n\right\} \cup\left\{ \pm 2 e_{i}: 1 \leq i \leq n\right\}, \text { and } \\
\mathfrak{f}_{4}: \Delta & = \pm\left\{e_{i}(i=1, \ldots, 4) ; e_{i} \pm e_{j}(1 \leq i<j \leq 4) ; \frac{1}{2}\left(e_{1} \pm e_{2} \pm e_{3} \pm e_{4}\right)\right\}
\end{aligned}
$$

For each case, the root length squares are either 1 or 2 and the claim is clearly true for them. Finally when $\mathfrak{g}=\mathfrak{g}_{2}$, the root length squares are either 2 or 6 ,

$$
\Delta= \pm\left\{e_{1}-e_{2}, e_{2}-e_{3}, e_{1}-e_{3}, 2 e_{1}-e_{2}-e_{3}, 2 e_{2}-e_{1}-e_{3}, 2 e_{3}-e_{1}-e_{2}\right\}
$$


and the claim is also true. As a result $\alpha\left(e^{i \gamma} x\right) \in \mathbb{R}$ for all $\alpha \in \Delta$ so $e^{i \gamma} x \in \mathfrak{h}_{\mathbb{R}}=i \mathfrak{t}$. Similarly we have the same conclusion for $y$. Then clearly $\theta_{j}-\rho_{j}$ is a constant, $j=1, \ldots, \ell$.

Proof of Theorem 2.2. We first show that the first four statements are equivalent.

$(1) \Rightarrow(2)$ : We may assume that $x \in \mathfrak{h}$. We have $B_{\theta}(K \cdot x, x)=B_{\theta}(\pi(K \cdot x), x)$ and it is convex since $\pi(K \cdot x)$ is convex.

(2) $\Leftrightarrow(3)$ : Lemma 2.6.

(3) $\Rightarrow(4)$ : The case $x=0$ is trivial. For $x \neq 0$, we may assume that each component $x_{j} \neq 0$ in the expression $x=x_{1}+\cdots+x_{\ell} \in \mathfrak{g}$. Then apply Proposition 2.7 .

(4) $\Rightarrow$ (1): By Theorem 2.1.

(5) $\Rightarrow(2)$ : obvious.

(4) $\Rightarrow(5)$ : Let $y=y_{1}+\cdots+y_{\ell} \in \mathfrak{g}_{1}+\cdots+\mathfrak{g}_{\ell}$. Then

$$
B_{\theta}(K \cdot x, y)=B_{\theta}\left(K_{1} \cdot x_{1}, y_{1}\right)+\cdots+B_{\theta}\left(K_{1} \cdot x_{\ell}, y_{\ell}\right) .
$$

By (4) there exist $k_{j} \in K_{j}$ and $\theta_{j} \in \mathbb{R}$ so that $t_{j}:=e^{i \theta_{j}} \operatorname{Ad}\left(k_{j}\right) x_{j} \in \mathfrak{t}_{j}$ for each $j=1, \ldots, \ell$. Write

$$
y_{j}=y_{j}^{(1)}+i y_{j}^{(2)} \text {, }
$$

for $y_{j}^{(1)}, y_{j}^{(2)} \in \mathfrak{k}$. So

$$
\begin{aligned}
B_{\theta}\left(K_{j} \cdot x_{j}, y_{j}\right) & =e^{-i \theta_{j}} B_{\theta}\left(K \cdot t_{j}, y_{j}\right) \\
& =e^{-i \theta_{j}}\left\{B\left(\operatorname{Ad}\left(k_{j}\right) t_{j}, y_{j}^{(1)}\right)+i B\left(\operatorname{Ad}\left(k_{j}\right) t_{j}, y_{j}^{(2)}\right): k_{j} \in K_{j}\right\}
\end{aligned}
$$

which is convex by a result of Tam [2002]. Hence $B_{\theta}(K \cdot x, y)$ is a sum of convex sets and thus convex.

Remark 2.8. The second author conjectured (see [Tam 2001, Conjecture 4.1]) that for a normal $x \in \mathfrak{g}$ (semisimple), if $B_{\theta}(K \cdot x, x)$ is convex, then there is $\gamma \in \mathbb{R}$ such that $e^{i \gamma} x \in \mathfrak{t}$. It is not true in view of Theorem 2.2. Consider the semisimple $\mathfrak{g}:=\mathfrak{a}_{1} \times \mathfrak{a}_{1}$. To be concrete, let $\mathfrak{g}=\mathfrak{s l}_{2}(\mathbb{C}) \times \mathfrak{s l}_{2}(\mathbb{C})$ with $K=\mathrm{SU}(2) \oplus \mathrm{SU}(2)$. Consider the normal $x=\operatorname{diag}\left(x_{1},-x_{1}\right) \oplus \operatorname{diag}\left(x_{2},-x_{2}\right)$, where $x_{1}, x_{2} \in \mathbb{C}$. Then for $k=k_{1} \oplus k_{2} \in K$,

$$
\begin{aligned}
\operatorname{tr} k x k^{-1} x^{*}= & \operatorname{tr} k_{1} \operatorname{diag}\left(x_{1},-x_{1}\right) k_{1}^{-1} \operatorname{diag}\left(\bar{x}_{1},-\bar{x}_{1}\right) \\
& +\operatorname{tr} k_{2} \operatorname{diag}\left(x_{2},-x_{2}\right) k_{2}^{-1} \operatorname{diag}\left(\bar{x}_{2},-\bar{x}_{2}\right) .
\end{aligned}
$$

By Theorem 1.3 the set

$$
\left\{\operatorname{tr} k_{i} \operatorname{diag}\left(x_{i},-x_{i}\right) k_{1}^{-1} \operatorname{diag}\left(\bar{x}_{i},-\bar{x}_{i}\right): k_{i} \in \mathrm{SU}(n)\right\}
$$

is convex, $i=1$, 2, so $\left\{\operatorname{tr} k x k^{-1} x^{*}: k \in K\right\}$ is the sum of two convex sets and thus is convex. However, $x_{1}, x_{2}$ need not be collinear with 0 . 
By Proposition 2.4 (a) $B_{\theta}(K \cdot x, x)$ is symmetric about the real axis. For some simple Lie algebras, more symmetry occurs for $B_{\theta}(K \cdot x, x)$ if $x \in \mathfrak{g}$ is normal. Indeed the symmetry is also true for $B_{\theta}(K \cdot x, y)$ for each $y \in \mathfrak{g}$.

Proposition 2.9. Let $\mathfrak{g}$ be simple and of type $\mathfrak{b}_{\ell}, \mathfrak{c}_{\ell}, \mathfrak{d}_{\ell}(\ell$ even $), \mathfrak{g}_{2}, \mathfrak{f}_{4}, \mathfrak{e}_{7}$ and $\mathfrak{e}_{8}$. Let $x \in \mathfrak{g}$ be normal. The sets $\pi(K \cdot x) \subset \mathfrak{h}$ and $B_{\theta}(K \cdot x, y) \subset \mathbb{C}$ are symmetric about the origin for each $y \in \mathfrak{g}$.

Proof. We may assume that $x \in \mathfrak{h}$. The Weyl group $W$ contains -1 [Helgason 1978, p. 523] so the desired result follows.

It is known [Djoković and Tam 2003] that if $x \in \mathfrak{g}$ is normal, then $B_{\theta}(K \cdot x, y)$ is star-shaped with respect to the center 0 for each $y \in \mathfrak{g}$.

We do not know whether $\pi(K \cdot x)$ is star shaped or not and the following conjectures [Tam 2001] are still open.

Conjecture 2.10. Let $\mathfrak{g}$ be a complex semisimple Lie algebra. If $x, y \in \mathfrak{g}$, then $B_{\theta}(K \cdot x, y)$ is star-shaped with respect to the star center 0 .

Conjecture 2.11. Let $\mathfrak{g}$ be a complex semisimple Lie algebra. If $x \in \mathfrak{g}$, then $\pi(K \cdot x)$ is star-shaped with respect to the star center 0 .

We remark that these conjectures can be reduced to the simple cases. The cases $\mathfrak{a}_{\ell}(\ell \geq 1), \mathfrak{d}_{\ell}(\ell \geq 2), \mathfrak{e}_{6}, \mathfrak{e}_{7}$ for Conjecture 2.10 are true [Cheung and Tsing 1996; Djoković and Tam 2003].

\section{Added in proof}

The authors very recently proved Conjecture 2.11 affirmatively.

\section{References}

[Au-Yeung and Sing 1977] Y. H. Au-Yeung and F. Y. Sing, "A remark on the generalized numerical range of a normal matrix”, Glasgow Math. J. 18:2 (1977), 179-180. MR 56 \#8594 Zbl 0359.47001 [Au-Yeung and Tsing 1983] Y. H. Au-Yeung and N.-K. Tsing, "A conjecture of Marcus on the generalized numerical range”, Linear and Multilinear Algebra 14:3 (1983), 235-239. MR 85e:15029 Zbl 0521.15016

[Bebiano and Da Providência 1996] N. Bebiano and J. Da Providência, "Another proof of a conjecture of Marcus on the $c$-numerical range", Linear and Multilinear Algebra 41:1 (1996), 35-40. MR 97g:15029 Zbl 0871.15028

[Cheung and Tsing 1996] W.-S. Cheung and N.-K. Tsing, "The $C$-numerical range of matrices is star-shaped”, Linear and Multilinear Algebra 41:3 (1996), 245-250. MR 97k:15060 Zbl 0876. 15021

[Djoković and Tam 2003] D. Z. Djoković and T.-Y. Tam, "Some questions about semisimple Lie groups originating in matrix theory", Canad. Math. Bull. 46:3 (2003), 332-343. MR 2004g:22008 Zbl 1047.22013 
[Helgason 1978] S. Helgason, Differential geometry, Lie groups, and symmetric spaces, Pure and Applied Mathematics 80, Academic Press, New York, 1978. MR 80k:53081 Zbl 0451.53038

[Horn 1954] A. Horn, "Doubly stochastic matrices and the diagonal of a rotation matrix", Amer. J. Math. 76 (1954), 620-630. MR 16,105c Zbl 0055.24601

[Kostant 1973] B. Kostant, "On convexity, the Weyl group and the Iwasawa decomposition", Ann. Sci. École Norm. Sup. (4) 6 (1973), 413-455 (1974). MR 51 \#806 Zbl 0293.22019

[Marcus 1979] M. Marcus, "Some combinatorial aspects of numerical range", Ann. New York Acad. Sci. 319 (1979), 368-376. MR 81g:15028 Zbl 0483.15017

[Schur 1923] I. Schur, "Über eine Klasse von Mittelbildungen mit Anwendungen auf der Determinantentheorie”, Sitzungsber. Berl. Math. Ges. 22 (1923), 9-20. JFM 49.0054.01

[Tam 2001] T.-Y. Tam, "On the shape of numerical ranges associated with Lie groups", Taiwanese J. Math. 5:3 (2001), 497-506. MR 2002f:15043 Zbl 0986.15022

[Tam 2002] T.-Y. Tam, "Convexity of generalized numerical range associated with a compact Lie group”, J. Aust. Math. Soc. 72:1 (2002), 57-66. MR 2002g:15056 Zbl 1007.15021

[Tsing 1981] N. K. Tsing, "On the shape of the generalized numerical ranges", Linear and Multilinear Algebra 10:3 (1981), 173-182. MR 82m:15026 Zbl 0471.47002

[Tsing 1984] N.-K. Tsing, "The constrained bilinear form and the $C$-numerical range", Linear Algebra Appl. 56 (1984), 195-206. MR 85b:15033 Zbl 0528.15007

[Westwick 1975] R. Westwick, "A theorem on numerical range", Linear and Multilinear Algebra 2 (1975), 311-315. MR 51 \#11132 Zbl 0303.47001

Received December 15, 2007. Revised February 29, 2008.

TIN-YAU TAM

DEPARTMENT OF MATHEMATiCS AND STATistics

221 PARKER HALL

AUBURN UNIVERSITY

AUBURN, AL 36849-5310

UNITED STATES

tamtiny@auburn.edu

WAI-SHUN CHEUNG

DePartment of MATHEMATiCs and Statistics

221 PARKER HALL

AUBURN UNIVERSITY

AUBURN, AL 36849-5310

UNITED STATES

cheungwaishun@gmail.com 